\title{
Optimization of the lidar optical design for measurement of the aerosol extinction vertical profile
}

\author{
Alessia Sannino ${ }^{1}$, Antonella Boselli ${ }^{2}$, Gaetano $\mathrm{Sasso}^{3}$, Nicola Spinelli ${ }^{1}$, Xuan Wang ${ }^{4 *}$ \\ ${ }^{1}$ Consorzio Nazionale Interuniversitario per le Scienze Fisiche della Materia (CNISM) and Dip. Fisica "E. Pancini", \\ Università degli Studi di Napoli Federico II, Napoli, Italy \\ ${ }^{2}$ Istituto di Metodologie per l'Analisi Ambientale (IMAA) - CNR, Italy \\ ${ }^{3}$ ALA Advanced lidar Applications s.r.l., Napoli, Italy \\ ${ }^{4}$ Istituto Superconduttori, Materiali innovativi e Dispositivi (SPIN) - CNR, Italy
}

\begin{abstract}
A lidar for aerosol monitoring with conventional optical design can provide good quality signals from several hundred meters up to tens of kilometres above the ground, but the aerosol load is mainly contained (up to $80 \%$ ) in the planetary boundary layer that can have a height of only hundreds of meters above the ground level. Therefore, the measurement of the complete aerosol extinction profile is generally a very difficult challenge. In this paper, we proposed an optical design of lidar systems able of producing signals starting from a few tens of meters above the ground. The overlap profiles obtained from an optimized lidar was compared with ray tracing simulations and further conventional lidar apparatuses.
\end{abstract}

\section{Introduction}

lidar (Light Detention and Ranging) technology is a performing remote sensing application, able to provide range and time resolved measurements of the backscattered (or re-emitted after a stimulation) light from a target invested by pulsed laser light.

Due to their versatility, lidar systems are widely used in different contexts ranging from physics, Earth study to archaeology, automation of vehicle and forest survey. In particular, in the field of atmospheric studies, lidar is an optimal tool for the retrieval of aerosol particles' optical characteristics due to the relation between the lidar signal intensity and the backscattering and extinction coefficients.

Unfortunately, it is not possible to record the optical signal corresponding to the entire spatial range probed by the laser beam because of limitations due to a series of optical and geometric parameters of the device. These limitations include the divergence and the transverse dimension of the laser beam, the distance between laser beam and the axis of the receiving telescope, and the specific design of the receiving optics, in which the field of view and the f-number of the telescope have a particularly critical role.

All these contributions can be synthesized in a function of the sounded range, called overlap function (OF), describing the overlap between the outgoing laser beam and the receiver field of view in the range domain. In many cases the form of this function can be derived, at least in part, from simulations or specific calibration measures to correct the raw data, but this correction adds an uncertainty on the signal that becomes unacceptable at very short range. [1]
Usually the most performing lidars have an OF which becomes equal to 1 (full overlap) only at a height higher than $800 \mathrm{~m}$, thus rejecting, in ground-based lidar systems, the information from the atmospheric part with the highest density of aerosols and aerosol processes.

The uncertainty introduced by the correction of the OF, if possible, prevents in fact the inversion of the data lidar for the purpose of obtaining the optical parameters and the microphysical characteristics of the aerosols at low altitude. $[2,3,4]$

In this paper a new optical design of lidar systems has been considered by using ray tracing simulations to determine the overlap function profiles. The performing configuration system has been implemented in a prototype developed within the SLIDE (Small LIdar DEvices) project of the China-Italy Laser Remote Sensing Joint Research Centre, a cooperation between CNISM, the Beijing Research Institute of Telemetry (PRC) and ALA srl. Simulated and measured OF with Raman and horizontal pointing methods were also studied. The overall performances of the prototype have been studied and compared with those of two lidar systems of the Earlinet international network [5].

\section{Results}

In order to obtain a geometrical configuration of the system that maximizes the area of overlap between the laser beam and the telescope FOV, with the aim to extend the sounded range and reduce, as far as possible, the minimum observable height, simulations of the optical system have been carried out

\footnotetext{
* Corresponding author: xuan.wang@spin.cnr.it
} 
With this goal, we choose a monostatic configuration of the system using a secondary mirror with reduced dimension and a larger telescope FOV allowing the laser beam to lap over the mirror shadow at lower altitude. In addition, the small size of the secondary mirror reduces signal loss due to its shadow on the primary. Moreover, to reduce the minimum observable height a short focal length was considered.

Finally, the choice of a laser source with high repetition rate allowed improving the dynamic range of the detected signal and the accuracy of the lidar measurement avoiding saturation of the signal at lower altitude.

The most performing simulated lidar system has been realized in SLIDE with a very compact and portable design.

The system has a monostatic configuration, it is provided of a Ritchey-Crétien telescope with a primary mirror of $200 \mathrm{~mm}$ diameter and a small size secondary mirror of $54 \mathrm{~mm}$ for less information loss due to its shadow on the primary. In order to obtain a short focal length and to get better signals in the near range, the dimensions of the telescope were left unaltered, except that a lower fnumber equal to 2.5 instead of 4 was chosen and a larger field of view, FOV $=2 \mathrm{mrad}$, was selected. In fact, this value is ten times larger than the laser divergence $(0.2 \mathrm{mrad})$, instead of the common choice of two to five times. The lidar works at $355 \mathrm{~nm}$ with an elastic and a Raman channel receiver. For elastic returns, it has been equipped with parallel and cross polarization channels. The lidar is equipped with a scanning system necessary for the subsequent operations of the overlap function calibration. The overlap obtained has been compared with a simulated one to test the performance.

\subsection{Comparison with simulated Overlap function}

Both Raman and horizontal measurements have been used to obtain a reliable measurement of the OF with two different independent methods [6,7]. The comparison was made using a simulated $\mathrm{OF}\left(\mathrm{OF}_{\mathrm{s}}\right)$ obtained with a ray tracing tool. The $\mathrm{OF}_{\mathrm{s}}$ has been obtained with the known optical parameters of the system and the assumption of clean air and a Gaussian laser beam, perfectly superimposed with the telescope axis. The measurement of the OF with the horizontal pointing method was carried out in conditions of a stable and relatively homogeneous atmosphere. The Raman measurement of the OF requires the simultaneous determination of the vertical profile of the backscattering coefficient from the elastic signal alone, using Klett's [8] method, and the combination of the elastic and the Raman signals from $\mathrm{N}_{2}[9,10]$. These measurements have been obtained in night conditions and with particularly clean atmosphere. In the Raman-Elastic method application, the value chosen for the lidar Ratio (ratio between extinction and backscattering coefficients) at low altitude and used for the application of the Klett method, is the seasonal average value determined from systematic measurements of the Earlinet network at the Naples site.

The comparison of the simulated with two experimentally obtained OFs is shown in Fig. 1. It should be highlighted that the OF reaches unity value at very low altitudes. Both the Raman and the horizontal methods show values of the full overlap around $120 \mathrm{~m}$, in agreement with the simulation. It should be noted that the plotted signals have different range resolutions, $15 \mathrm{~m}$ and $60 \mathrm{~m}$, respectively for ray tracing and both the measured one.

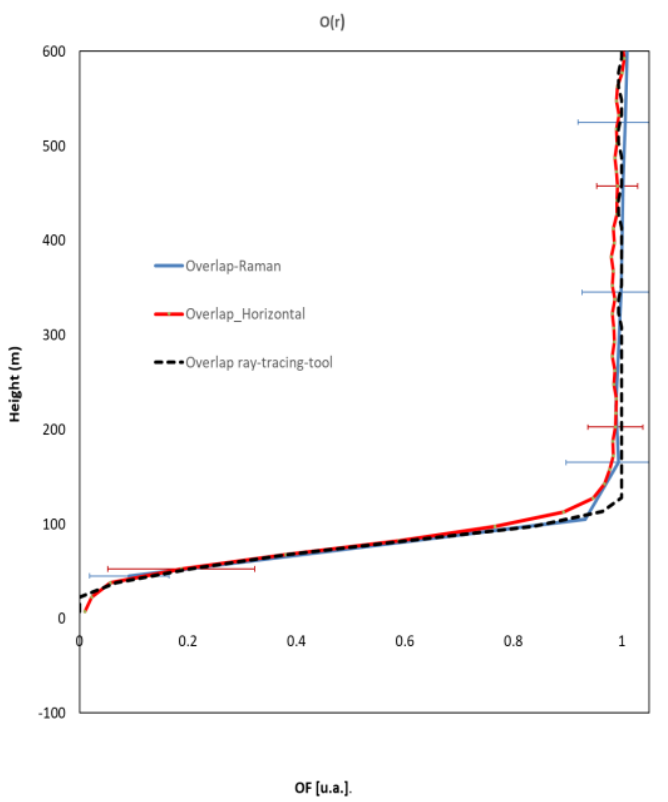

Fig. 1. Overlap function retrieved by the ray-tracing tool with range resolution of $15 \mathrm{~m}$ (dashed black), Raman method, with a range resolution of $60 \mathrm{~m}$ (blue), and relative error bars, and the horizontal method (red) with a range resolution of $15 \mathrm{~m}$ and relative error bars.

\subsection{Comparison of the signals measured with different lidar systems}

A comparison was made between the signals of the lidar developed within the SLIDE project with two lidar devices available at the Naples site of the Earlinet network. The first lidar system used is MALIA (Multiwavelength Aerosol LIdar Apparatus) [11,12], a multi wavelength fixed system based at the Physics Department of University "Federico II" di Napoli. MALIA is the main device for the systematic measurements of the Earlinet network. The receiving system of MALIA is based on a $30 \mathrm{~cm}$ Newtonian telescope with a focal length of $120 \mathrm{~cm}$, a FOV of $1.33 \mathrm{mrad}$

The second instrument is a compact UV lidar by Raymetrics (LR111-ESS-D200), recently installed as 
part of the strengthening of the site with the I-AMICA (http://www.i-amica.it) project of the CNR. It is used mainly for H24 measurements of special events and as back-up of the main system.

The LR111's optical scheme is based on a Cassegrain telescope with f-number 4 and a focal length of $800 \mathrm{~mm}$. The primary mirror is $200 \mathrm{~mm}$ diameter and a secondary $48 \mathrm{~mm}$.

The lidar is designed to acquire the signal in two modes: analog and photocounting. This characteristic is useful to obtain a more accurate signal; in fact, the acquisition in analogue mode is optimized to collect the signal at low altitudes while the photocounting mode is used for the higher ranges.

A comparison of the $\mathrm{N}_{2}$ Raman signals at $386 \mathrm{~nm}$ for MALIA and SLIDE lidars is reported in Fig. 2. The figure shows the range corrected signal (RCS), without correction for the overlap function. Both signals are acquired in the photocounting regime.

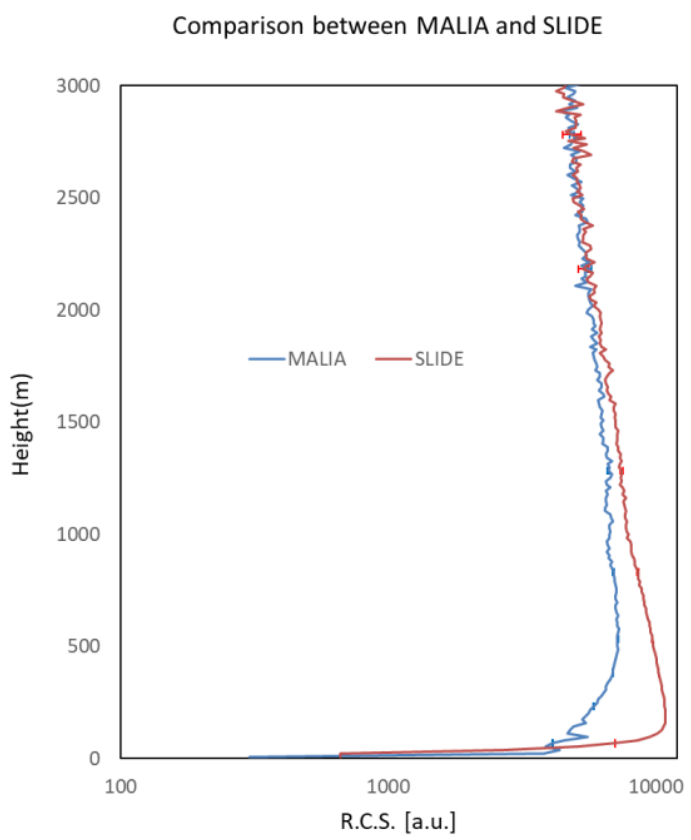

Fig. 2 Range Corrected Signal (R.C.S.) comparison between MALIA (blue) and the SLIDE lidar prototype (red) for the $386 \mathrm{~nm}$ digital channel. The statistical errors are of the order of $0.5 \%$ up to $500 \mathrm{~m}$ and $3 \%$ at $1500 \mathrm{~m}$.

Fig. 3 shows the comparison between elastic signals at $355 \mathrm{~nm}$, with the same linear polarization as the laser beam, from SLIDE and the LR111 device. It should be noted that LR111 uses two distinct detection channels for the high and low altitudes to increase the signal dynamics and lower the full overlap height without worsening the signal to noise ratio at high altitudes. Then the LR111 signal shown in the figure is the result of merging the two signals. The SLIDE lidar signal shown in the same figure is obtained with the use of a single detector operating in photocounting mode.
Comparison between Raymetrics LR111-ESS-D200 and SLIDE

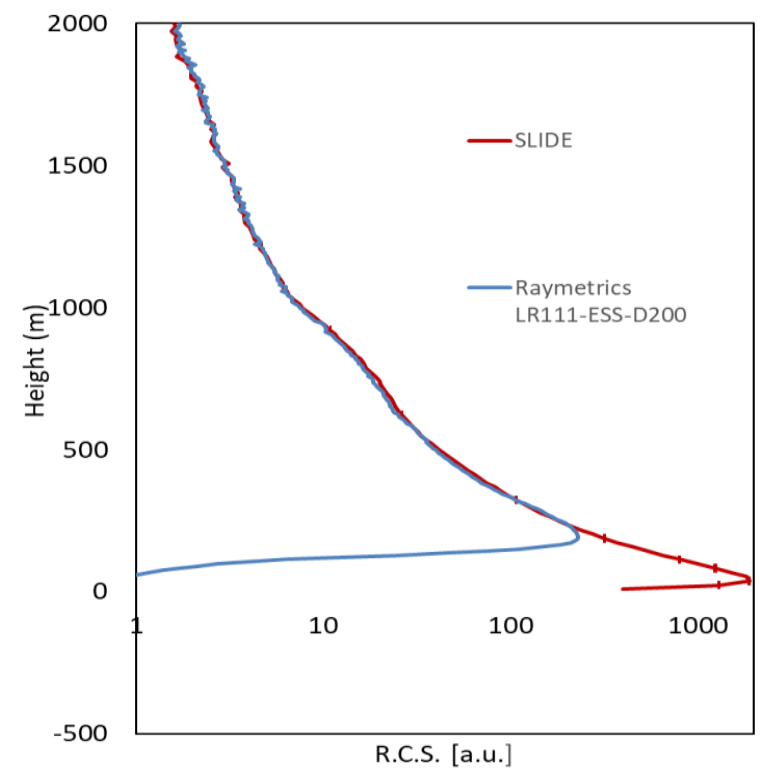

Fig. 3 LR111 (red) and the SLIDE lidar p (blue) comparison for the Range Corrected Signal (R.C.S.) at $355 \mathrm{~nm}$ with parallel polarization. The red line is obtained by merging data from the analog and photocounting detection channels. The statistical errors are of the order of $0.5 \%$ up to $500 \mathrm{~m}$ and $3 \%$ at $1500 \mathrm{~m}$.

\section{Conclusions}

A careful design of the optical assembly of a lidar system has been conducted in order to find the best configuration which guarantees a useful signal starting from the lowest possible altitude. In particular, the study involved three optical parameters: larger FOV, smaller diameter of the secondary mirror and a reduced fnumber. The optimized optical system has been designed preserving a relatively small telescope size $(200 \mathrm{~mm})$ but with f-number of 2.5 instead of more than 4 , in order to obtain a short focal length. in addition, the optical design allows the use of a very small (54 mm diameter) secondary mirror in order to reduce its shadowing effect on OF. At last we have chosen a larger FOV of $2 \mathrm{mrad}$ respect to the $0.2 \mathrm{mrad}$ of the laser divergence.

This optimized optical system has been realized for a compact lidar system which allowed to test its overlap function both with simulated and measured results. The test on overlap function both with simulations and measurements show that the OF reaches the unity value (full overlap) at $120 \mathrm{~m}$ and an effective correction for the OF can be performed down to $30 \mathrm{~m}$ height. 


\section{References}

1. G. Biavati, G. Di Donfrancesco, F. Cairo, and D. G. Feist. Correction scheme for close-range lidar returns. Applied Optics, 50 (30), 5872-5882, 2011. 27, 2011.

2. Moritz Haarig, Albert Ansmann, Dietrich Althausen, André Klepel, Silke Groß, Volker Freudenthaler, Carlos Toledano, Rodanthi-Elisavet Mamouri, David A. Farrell, Damien A. Prescod, Eleni Marinou, Triple-wavelength depolarization-ratio profiling of Saharan dust over Barbados during SALTRACE in 2013 and 2014, Atmos. Chem. Phys., 17, 1076710794, 2017 https://doi.org/10.5194/acp-17-107672017

3. Groß, S., Gasteiger, J., Freudenthaler, V., Müller, T., Sauer, D., Toledano, C., and Ansmann, A.: Saharan dust contribution to the Caribbean summertime boundary layer - a lidar study during SALTRACE, Atmos. Chem. Phys., 16, 11535-11546, https://doi.org/10.5194/acp-16-11535-2016, 2016.

4. C. Weitekamp, "lidar Range-Resolved Optical Remote Sensing of the Atmosphere", Singapore: Springer; 2005

5. Jens Bösenberg et al, "EARLINET: A European Aerosol Research lidar Network to Establish an Aerosol Climatology", Hamburg, September 2003

6. X. Wang, A. Boselli, A.Sannino, C. Song, N. Spinelli, Y. Zhao and C. Pan "Calibration of Multiwavelength Raman Polarization lidar.” EPJ Web of Conferences, $89 \quad$ (2015) $01002 \quad$ DOI: https://doi.org/10.1051/epjconf/20158901002

7. Wandinger U, Ansmann A. Experimental determination of the lidar overlap profile with Raman lidar. Appl Opt. 2002 Jan 20;41(3):511-4. PubMed PMID: 11905578.

8. Klett J. Stable analytic inversion solution for processing lidar returns. Appl. Opt., vol. 20, No.2, 1981.

9. Albert Ansmann, et al. Indipendent measurements of extinction and backscatter profiles in cirrus clouds by using a combined Raman elastic-backscatter LIDAR - Applied Optics vol 31, n.33 (1992)

10. Ansmann and Wandinger, Combined Raman Elastic Backscatter LIDAR for vertical profiling of moisture, aerosol extinction, backscatter and lidar ratio Applied Physic, B55, 18-28 (1992).

11. G. Pisani "lidar study of high density aerosol clouds: the Aerosol Multi-wavelength Polarization lidar Experiment." PhD Thesis Università Federico II di Napoli 2013.

12. L. Mona C. Cornacchia, G. D'Amico, P. Di Girolamo, G. Pappalardo, G. Pisani, D. Summa, X. Wang and V. Cuomo, Characterization of the variability of the humidity and cloud fields as observed from a cluster of ground-based lidar systems, QUARTERLY JOURNAL OF THE ROYAL METEOROLOGICAL SOCIETY Q. J. R. Meteorol. Soc. 133: (S3) 257-271 (2007) Published online in Wiley InterScience (www.interscience.wiley.com) DOI: 10.1002/qj.160. 